\title{
On the infimum attained by the reflected fractional Brownian motion
}

\author{
K. Dębicki • K. M. Kosiński
}

Received: 5 October 2013 / Revised: 28 March 2014 /

Accepted: 7 April 2014 / Published online: 12 July 2014

(C) The Author(s) 2014. This article is published with open access at Springerlink.com

Abstract Let $\left\{B_{H}(t): t \geq 0\right\}$ be a fractional Brownian motion with Hurst parameter $H \in\left(\frac{1}{2}, 1\right)$. For the storage process $Q_{B_{H}}(t)=\sup _{-\infty \leq s \leq t}$ $\left(B_{H}(t)-B_{H}(s)-c(t-s)\right)$ we show that, for any $T(u)>0$ such that $T(u)=$ $o\left(u^{\frac{2 H-1}{H}}\right)$,

$$
\mathbb{P}\left(\inf _{s \in[0, T(u)]} Q_{B_{H}}(s)>u\right) \sim \mathbb{P}\left(Q_{B_{H}}(0)>u\right),
$$

as $u \rightarrow \infty$. This finding, known in the literature as the strong Piterbarg property, goes in line with previously observed properties of storage processes with self-similar and infinitely divisible input without Gaussian component.

Keywords Extremes of Gaussian fields · Storage processes · Fractional Brownian motion

AMS 2000 Subject Classifications Primary: 60F15 - 60G70; Secondary: 60G22

\footnotetext{
K. Dȩbicki

Mathematical Institute, University of Wrocław, pl. Grunwaldzki 2/4, 50-384 Wrocław, Poland e-mail: Debicki@math.uni.wroc.pl

K. M. Kosiński $(\square)$

Institute of Mathematics, University of Warsaw, ul. Banacha 2, 02-097 Warsaw, Poland e-mail: Kosinski@mimuw.edu.pl
} 


\section{Introduction}

The analysis of distributional properties of reflected stochastic processes is continuously motivated both by theory- and applied-oriented open problems in probability theory. In this paper we analyze the asymptotic properties of tail distribution of infimum of an important class of such processes, that naturally appear in models of storage (queueing) systems and, by duality to ruin problems, gained broad interest also in problems arising in finance and insurance risk; see, e.g., Norros (2004), Piterbarg (2001), Asmussen (2003), and Asmussen and Albrecher (2010) or a novel work (Hashorva et al. 2013).

Consider a fluid queue with infinite buffer capacity, service rate $c>0$ and the total inflow by time $t$ modeled by a stochastic process with stationary increments $X=\{X(t): t \in \mathbb{R}\}$. Following Reich (1958), the stationary storage process that describes the stationary buffer content process, has the following representation

$$
Q_{X}(t)=\sup _{-\infty \leq s \leq t}(X(t)-X(s)-c(t-s)) .
$$

There is a strong motivation for modeling the input process $X$ by a fractional Brownian motion (fBm) $B_{H}=\left\{B_{H}(t): t \in \mathbb{R}\right\}$ with $H>1 / 2$, i.e., a centered Gaussian process with stationary increments, continuous sample paths a.s., and variance function $\sigma_{B_{H}}^{2}(t)=t^{2 H}$. On one hand, such structural properties of fBm as self-similarity and long range dependence, have been statistically confirmed in data analysis of many real traffic processes in modern data-transfer networks. On the other hand, in Taqqu et al. (1997) and Mikosch et al. (2002) it was proven that appropriately scaled aggregation of large number of (integrated) On-Off input processes with regularly varying tail distribution of successive On-times, converges to an fBm with $H>1 / 2$.

The importance of $\mathrm{fBm}$ storage processes resulted in a vast interest of analysis of the process $Q_{B_{H}}$. In particular finding the properties of finite-dimensional (or at least 1-dimensional) distributions of $Q_{B_{H}}$ has been a long standing goal; see Norros (2004) and Piterbarg (2001). The stationarity of increments of $B_{H}$ implies the stationarity of the process $Q_{B_{H}}$, so that, for any fixed $t$, the random variable $Q_{B_{H}}(t)$ has the same distribution as $Q_{B_{H}}(0)$. Nevertheless, apart from the Brownian case $H=\frac{1}{2}$, the exact distribution of $Q_{B_{H}}(0)$ is not known. Therefore, one usually resorts to the exact asymptotics of $\mathbb{P}\left(Q_{B_{H}}(0)>u\right)$, as $u \rightarrow \infty$. These have been found for the full range of parameter $H \in(0,1)$ in Hüsler and Piterbarg (1999), leading to,

$$
\mathbb{P}\left(Q_{B_{H}}(0)>u\right) \sim \sqrt{\pi} a^{\frac{1}{2 H}} b^{-\frac{1}{2}} \mathcal{H}_{B_{H}}^{\text {sup }}\left(A u^{1-H}\right)^{\frac{1-H}{H}} \Psi\left(A u^{1-H}\right) \text {, as } u \rightarrow \infty,
$$

where the constants $a, b$ and $A$ can be given explicitly (see Section 4 ), $\mathcal{H}_{B_{H}}^{\text {sup }}$ is the so-called Pickands constant, and $\Psi(u)$ denotes the right tail of the standard normal distribution.

Piterbarg (2001) considered the supremum of the process $Q_{B_{H}}$ on the interval $[0, T]$ and found the exact asymptotics of

$$
\mathbb{P}\left(\sup _{t \in[0, T]} Q_{B_{H}}(t)>u\right), \quad \text { as } u \rightarrow \infty
$$


for the whole range of the parameter $H$. By comparing them with Eq. 1, he observed a remarkable property that, for $H>\frac{1}{2}$, and any positive function $T=T(u)$ such that $T(u)=o\left(u^{\frac{2 H-1}{H}}\right)$,

$$
\mathbb{P}\left(\sup _{t \in[0, T]} Q_{B_{H}}(t)>u\right) \sim \mathbb{P}\left(Q_{B_{H}}(0)>u\right), \quad \text { as } u \rightarrow \infty .
$$

This property is nowadays referred to as the generalized Piterbarg property; see Albin and Samorodnitsky (2004). As a corollary from Eq. 2 one easily gets that for any fixed $n>0$ and $t_{1}, \ldots, t_{n} \in[0, T]$, with $u \rightarrow \infty$,

$\mathbb{P}\left(\min _{i=1, \ldots, n} Q_{B_{H}}\left(t_{i}\right)>u \mid \sup _{t \in[0, T]} Q_{B_{H}}(t)>u\right) \geq 1-\sum_{i=1}^{n}\left(1-\frac{\mathbb{P}\left(Q_{B_{H}}\left(t_{i}\right)>u\right)}{\mathbb{P}\left(\sup _{t \in[0, T]} Q_{B_{H}}(t)>u\right)}\right) \rightarrow 1$.

This leads to the natural question, whether the minimum over finite number of points can be substituted with the infimum functional, which then leads to

$$
\mathbb{P}\left(\inf _{t \in[0, T]} Q_{B_{H}}(t)>u\right) \sim \mathbb{P}\left(\sup _{t \in[0, T]} Q_{B_{H}}(t)>u\right), \quad \text { as } u \rightarrow \infty .
$$

This property shall be referred to as the strong Piterbarg property.

The above terminology has been coined by Albin and Samorodnitsky (2004), who, motivated by Piterbarg (2001), considered the case when the input process $X$ belongs to the class of self-similar infinitely divisible stochastic processes with no Gaussian component. They provide general conditions under which Eqs. 2 and 3 hold with $Q_{X}$ instead of $Q_{B_{H}}$. The approach in Albin and Samorodnitsky (2004) is based on the assumption that the Lévy measure associated with $X$ has heavy tails, which combined with the absence of a Gaussian component allows for more direct and less delicate methods to be employed. It is the light-tailed nature of the Gaussian distribution that renders the problem of the asymptotics of suprema of Gaussian processes hard. Furthermore, infima of Gaussian processes (apart perhaps from the Brownian case) have not been considered systematically. On the high level, the problem stems from the fact that an infimum is, by definition, an intersection of events. If the number of events grows to infinity, then the intersection is much harder to handle than, for instance, the sum of events (which corresponds to the supremum).

In this paper we derive exact asymptotics of

$$
\mathbb{P}\left(\inf _{t \in[0, T]} Q_{B_{H}}(t)>u\right), \quad \text { as } u \rightarrow \infty .
$$

and prove that the strong Piterbarg property (3) holds for the same range of functions $T(u)$ as in the generalized Piterbrag property (2), i.e., $T(u)=o\left(u^{\frac{2 H-1}{H}}\right), H>\frac{1}{2}$. The idea of the proof is based on finding the exact asymptotics of

$$
\mathbb{P}\left(\Phi\left(X_{u}\right)>u\right), \quad \text { as } u \rightarrow \infty,
$$

for a broad class of continuous functionals $\Phi: C(T) \rightarrow \mathbb{R}$ acting on the space $C(T)$ of continuous functions on compacts $T \subset \mathbb{R}_{+}^{d}, d \geq 1$, and a broad class of Gaussian fields $X_{u}=\left\{X_{u}(\mathbf{t}): \mathbf{t} \in \mathbb{R}_{+}^{d}\right\}$. The connection between Eqs. 4 and 5 can be seen 
by setting $d=1, \Phi(f)=\inf _{t \in[0,1]} f(t)$ and $X_{u}(t)=Q_{B_{H}}(T(u) t)$, although the relation is far from straight forward since $Q_{B_{H}}$ is not Gaussian.

Structure of the paper The exact asymptotics of Eq. 5 are given in Lemma 1 (see Section 3), which is the first contribution of this paper. Interestingly, the asymptotics of Eq. 5 involve a new type of constants of the form

$$
\mathcal{H}_{\eta}^{\Phi}(T)=\mathbb{E} \exp \left(\Phi\left(\sqrt{2} \eta(\cdot)-\sigma_{\eta}^{2}(\cdot)\right)\right),
$$

where $\eta$ is a Gaussian random field with variance function $\sigma_{\eta}^{2}$. These new constants extend the notion of the classical Pickands' constants $\mathcal{H}_{B_{H}}^{\text {sup }}(S)=$ $\mathbb{E} \exp \left(\sup _{t \in[0, S]}\left(\sqrt{2} B_{H}(t)-t^{2 H}\right)\right), S>0$, dating back to Pickands (1969b). Recall that $\mathcal{H}_{B_{H}}^{\text {sup }}=\lim _{S \rightarrow \infty} \mathcal{H}_{B_{H}}^{\text {sup }}([0, S]) / S$ in Eq. 1. In Theorem 1 (Section 4) we give the strong Piterbarg property, which is the second contribution of this paper. More precisely, we show that Eq. 3 holds for $H>\frac{1}{2}$ and $T(u)=o\left(u^{\frac{2 H-1}{H}}\right)$, i.e., the same order of functions for which Eq. 2 holds. In Sections 5 and 6 we give the proofs of our main results.

\section{Notation}

Before we begin, let us set the notation that will be used throughout the paper. By $B_{H}=\left\{B_{H}(t): t \in \mathbb{R}\right\}$ we denote the fBm with Hurst parameter $H \in(0,1)$, that is, a Gaussian process with zero mean and covariance function given by

$$
\operatorname{Cov}\left(B_{H}(t), B_{H}(s)\right)=\frac{1}{2}\left(|t|^{2 H}+|s|^{2 H}-|t-s|^{2 H}\right) .
$$

Let $\Psi$ be the right tail of the standard normal distribution. Recall that

$$
\Psi(u)=\frac{1}{u \sqrt{2 \pi}} \exp \left(-\frac{u^{2}}{2}\right)\left(1+O\left(u^{-2}\right)\right), \quad \text { as } u \rightarrow \infty .
$$

For any vector $\mathbf{t} \in \mathbb{R}^{d}, d \geq 1$, we denote $\mathbf{t}=\left(t_{1}, \ldots, t_{n}\right)$. By $\eta=\left\{\eta(\mathbf{t}): \mathbf{t} \in \mathbb{R}_{+}^{d}\right\}$ we denote a centered Gaussian field, with almost surely sample paths, $\eta(0)=0$ and variance function $\sigma_{\eta}^{2}(\mathbf{t})=\operatorname{Var}(\eta(\mathbf{t}))$. Let us introduce the following condition:

E1: $\mathbb{E}\left(\eta\left(\mathbf{t}_{1}\right)-\eta\left(\mathbf{t}_{2}\right)\right)^{2} \leq G\left\|\mathbf{t}_{1}-\mathbf{t}_{2}\right\|^{\gamma}$, for some $\gamma, G>0$ and every $\mathbf{t}_{1}, \mathbf{t}_{2} \in \mathbb{R}_{+}^{d}$.

Condition E1 is a standard regularity requirement; see, e.g., Piterbarg (1996). Now let $\Phi: C(T) \rightarrow \mathbb{R}$ be a continuous functional acting on $C(T)$, the space of continuous functions on compacts $T \subset \mathbb{R}_{+}^{d}, d \geq 1$. Assume that:

F1: $|\Phi(f)| \leq \sup _{\mathbf{t} \in T} f(\mathbf{t})$,

F2: $\Phi(a f+b)=a \Phi(f)+b$, for every $a, b>0$.

For $\Phi$ satisfying $\mathbf{F} \mathbf{1}$ we define a constant $\mathcal{H}_{\eta}^{\Phi}(T)$ via

$$
\mathcal{H}_{\eta}^{\Phi}(T)=\mathbb{E} \exp \left(\Phi\left(\sqrt{2} \eta(\cdot)-\sigma_{\eta}^{2}(\cdot)\right)\right) .
$$


Note that the dependence on $T$ is implicit via $\Phi: C(T) \rightarrow \mathbb{R}$. To see that the above constant is well defined, notice that due to $\mathbf{F 1}, \mathbb{P}\left(\Phi\left(\sqrt{2} \eta(\cdot)-\sigma_{\eta}^{2}(\cdot)\right)>u\right) \leq$ $\mathbb{P}\left(\sup _{\mathbf{t} \in T} \eta(\mathbf{t})>u / \sqrt{2}\right)$. Now since $\eta$ is continuous, then it has bounded sample paths a.s. and $\sigma_{\eta}^{2}=\sup _{\mathbf{t} \in T} \sigma_{\eta}^{2}(\mathbf{t})<\infty$. Let $m=\mathbb{E} \sup _{\mathbf{t} \in T} \eta(\mathbf{t})$. Borell's inequality; see, e.g., Adler (1990), implies that for $x>m$, $\mathbb{P}\left(\sup _{\mathbf{t} \in T} \eta(\mathbf{t})>x\right) \leq 2 \exp \left(-(x-m)^{2} /\left(2 \sigma_{\eta}^{2}\right)\right)$ and, as a consequence, $\mathcal{H}_{\eta}^{\Phi}(T)=$ $\int_{-\infty}^{\infty} e^{x} \mathbb{P}\left(\Phi\left(\sqrt{2} \eta(\cdot)-\sigma_{\eta}^{2}(\cdot)\right)>x\right) \mathrm{d} x<\infty$.

\section{Generalized Pickands' lemma}

In this section we present a lemma that shall play a crucial role in proving the strong Piterbarg property in the remaining part of the paper.

Let us recall that the original Pickands' lemma (Pickands 1969a, 1969b) concerns with a stationary Gaussian process $X$ with zero mean and covariance function $r(t)$ satisfying $r(t)=1-|t|^{2 H}+o\left(|t|^{2 H}\right)$, as $t \rightarrow 0$, for some $H \in(0,1)$, and $r(t)<1$ for all $t>0$. Its conclusion states that, for any $S>0$,

$$
\mathbb{P}\left(\sup _{t \in[0, S]} X_{u}(t)>u\right) \sim \mathcal{H}_{B_{H}}^{\text {sup }}([0, S]) \Psi(u), \quad \text { as } u \rightarrow \infty,
$$

where $X_{u}(t)=X\left(t u^{-1 / H}\right)$. Pickands' lemma has been generalized in various ways, capturing both nonstationarity of $X$ and extension to Gaussian fields; see, e.g., Piterbarg (1996). Dȩbicki (2002) presented an extension covering broader local covariance structures, than satisfying $\operatorname{Cov}(X(s), X(t))=1-|s-t|^{\alpha}+o\left(|s-t|^{\alpha}\right)$ as $s-t \rightarrow 0$, for some $\alpha \in(0,2]$. Among others, notable extensions have been recently considered in Dȩbicki and Tabiś (2011).

In the following lemma we present a version of Pickands' lemma that captures the new constant $\mathcal{H}_{\eta}^{\Phi}(T)$ introduced in the previous section.

Lemma 1 (Generalized Pickands' lemma) For any $u>0$, let $X_{u}=\left\{X_{u}(\mathbf{t}): \mathbf{t} \in\right.$ $\left.\mathbb{R}_{+}^{d}\right\}$ be a centered Gaussian field with a constant variance equal to one. Let the correlation function $r_{u}\left(\mathbf{t}_{1} ; \mathbf{t}_{2}\right)=\mathbb{C o r r}\left(X_{u}\left(\mathbf{t}_{1}\right), X_{u}\left(\mathbf{t}_{2}\right)\right)$ satisfy

$$
\lim _{u \rightarrow \infty} \sup _{\mathbf{t}_{1}, \mathbf{t}_{2} \in T}\left|\frac{f^{2}(u)\left(1-r_{u}\left(\mathbf{t}_{1} ; \mathbf{t}_{2}\right)\right)}{\operatorname{Var}\left(\eta\left(\mathbf{t}_{1}\right)-\eta\left(\mathbf{t}_{2}\right)\right)}-1\right|=0,
$$

for some compact set $T \subset \mathbb{R}_{+}^{d}$, some function $f(u) \rightarrow \infty$, as $u \rightarrow \infty$, and $\eta$ satisfying $\boldsymbol{E} 1$. Let $\Phi: C(T) \rightarrow \mathbb{R}$ be a continuous functional satisfying F1-F2. Then, for any function $n(u)$ such that $n(u) \sim f(u)$,

$$
\mathbb{P}\left(\Phi\left(X_{u}\right)>n(u)\right) \sim \mathcal{H}_{\eta}^{\Phi}(T) \Psi(n(u)), \quad \text { as } u \rightarrow \infty .
$$

Remark 1 Conditions similar to assumption (8) have been introduced in, among others, Dębicki (2002), Hüsler and Piterbarg (2004), Dębicki and Kisowski (2008), and Dębicki and Tabiś (2011) as a standard way of capturing nonstationarity. The shape 
of Lemma 1 is tailored to the needs of the next section, where asymptotics of tail distribution of inf sup functionals of Gaussian processes are analyzed. Various further extensions of Lemma 1 can be thought of along the lines of already existing extensions of the classical Pickands' lemma, especially in the direction allowing nonconstant variance function of the family $\left(X_{u}\right)$, as in Piterbarg and Prisyazhnyuk (1978) or Hashorva et al. (2013).

Example 1 Assume that $X=\left\{X(\mathbf{t}): \mathbf{t} \in \mathbb{R}_{+}^{d}\right\}$ is a centered Gaussian field with unit variance and function satisfying

$r\left(\mathbf{t}_{1} ; \mathbf{t}_{2}\right)=1-\sum_{i=1}^{d} a_{i}\left|t_{1, i}-t_{2, i}\right|^{2 H_{i}}+o\left(\sum_{i=1}^{d}\left|t_{1, i}-t_{2, i}\right|^{2 H_{i}}\right)$, as $\sum_{i=1}^{d}\left|t_{1, i}-t_{2, i}\right| \rightarrow 0$,

for some $H_{i} \in(0,1), a_{i}>0, i=1, \ldots, d$. Define a new field $X_{u}=\left\{X_{u}(\mathbf{t})\right.$ : $\left.\mathbf{t} \in \mathbb{R}_{+}^{d}\right\}$ via $X_{u}(\mathbf{t})=X\left(t_{1} u^{-\frac{1}{H_{1}}}, \ldots, t_{d} u^{-\frac{1}{H_{d}}}\right)$. For any compact set $T \subset \mathbb{R}_{+}^{d}$, the process $X_{u}$ satisfies (8) with $f(u)=u$ and $\boldsymbol{\eta}(\mathbf{t})=\sum_{i=1}^{d} B_{H_{i}}^{i}\left(a^{\frac{1}{2 H_{i}}} t_{i}\right)$, where $B_{H_{i}}^{i}$ constitute independent fBm's with Hurst parameters $H_{i}$. Hence the conclusion of Lemma 1 holds for any functional $\Phi$ on $C(T)$ satisfying F1-F2. In the following section we shall encounter this example in the setting of $d=2, H_{1}=H_{2}, a_{1}=a_{2}$ and $\Phi(f)=\inf _{t_{1} \in\left[0, \lambda_{1}\right]} \sup _{t_{2} \in\left[0, \lambda_{2}\right]} f(\mathbf{t})$, for some $\lambda_{1}, \lambda_{2}>0$. In this case, with $H=H_{1}$ and $a=a_{1}$, for any function $n(u) \sim u$,

$\mathbb{P}\left(\inf _{t_{1} \in\left[0, \lambda_{1}\right]} \sup _{t_{2} \in\left[0, \lambda_{2}\right]} X_{u}(\mathbf{t})>n(u)\right)=\mathcal{H}_{B_{H}}^{\inf }\left(\left[0, a^{\frac{1}{2 H}} \lambda_{1}\right]\right) \mathcal{H}_{B_{H}}^{\text {sup }}\left(\left[0, a^{\frac{1}{2 H}} \lambda_{2}\right]\right) \Psi(n(u))$, as $u \rightarrow \infty$.

\section{Strong Piterbarg property}

In this section we present the main result of this paper. Let us first recall the definition of the storage process $Q_{B_{H}}$ with service rate $c>0$ and input $B_{H}$,

$$
Q_{B_{H}}(t)=\sup _{-\infty \leq s \leq t}\left(B_{H}(t)-B_{H}(s)-c(t-s)\right) .
$$

Let us define the following constants: $a=\frac{1}{2} \tau_{0}^{-2 H}, b=\frac{B}{2 A}, A=\frac{1}{1-H} \tau_{0}^{-H}, B=$ $H \tau_{0}^{-H-2}, \tau_{0}=\frac{H}{c(1-H)}$, see Eq. 1. Finally, let

$$
\mathcal{H}_{B_{H}}^{\text {sup }}=\lim _{S \rightarrow \infty} \frac{\mathcal{H}_{B_{H}}^{\text {sup }}([0, S])}{S}
$$

be the classical Pickands's constant. Now we are in position to state our main result.

Theorem 1 (Strong Piterbarg property) For $H>\frac{1}{2}$ and any $T(u)>0$, such that $T(u)=o\left(u^{\frac{2 H-1}{H}}\right)$,

$\mathbb{P}\left(\inf _{t \in[0, T(u)]} Q_{B_{H}}(t)>u\right) \sim \sqrt{\pi} a^{\frac{1}{2 H}} b^{-\frac{1}{2}} \mathcal{H}_{B_{H}}^{\text {sup }} \cdot\left(A u^{1-H}\right)^{\frac{1-H}{H}} \Psi\left(A u^{1-H}\right)$, as $u \rightarrow \infty$. 
In particular,

$\mathbb{P}\left(\inf _{t \in[0, T(u)]} Q_{B_{H}}(t)>u\right) \sim \mathbb{P}\left(Q_{B_{H}}(0)>u\right) \sim \mathbb{P}\left(\sup _{t \in[0, T(u)]} Q_{B_{H}}(t)>u\right)$, as $u \rightarrow \infty$

Remark 2 The asymptotics of $\mathbb{P}\left(Q_{B_{H}}(0)>u\right)$ were found in (Hüsler and Piterbarg 1999, Theorem 1); cf. Eq. 1. The asymptotic equivalence between the tail decay of the supremum functional and the value of $Q_{B_{H}}$ at 0 was proven in (Piterbarg 2001, Theorem 5) and is called the Piterbarg property, as mentioned in the introduction; cf. Eq. 2. Note that the formula in (Piterbarg 2001, Theorem 5) should have $a^{\frac{1}{2 H}}$ as cited here instead of $a^{\frac{1}{H}}$.

Remark 3 The case of Brownian motion, that is $H=\frac{1}{2}$, has been treated in (Dębicki et al. 2012, Theorem 3). The authors found the exact distribution of the infimum of $Q_{B_{\frac{1}{2}}}$ attained on any interval of the form $[0, S], S>0$,

$$
\mathbb{P}\left(\inf _{t \in[0, S]} Q_{B_{\frac{1}{2}}}(t)>u\right)=\mathbb{P}\left(Q_{B_{\frac{1}{2}}}(0)>u\right)\left(2(1+S) \Psi(\sqrt{S})-\sqrt{\frac{2 S}{\pi}} \exp \left(-\frac{S}{2}\right)\right) .
$$

Let us recall that $Q_{B_{\frac{1}{2}}}(0)$ has $\frac{1}{2}$-exponential distribution. On the other hand, Piterbarg (2001, Theorem 6), gives (note that the original formula in Piterbarg 2001 has a misprint)

$$
\mathbb{P}\left(\sup _{t \in[0, S]} Q_{B_{\frac{1}{2}}}(t)>u\right) \sim \mathbb{P}\left(Q_{B_{\frac{1}{2}}}(0)>u\right) 2 \sqrt{\pi} \mathcal{H}_{B_{\frac{1}{2}}}^{\sup }([0,2 S]), \text { as } u \rightarrow \infty
$$

Therefore, we see that the strong Piterbarg property does not hold in the case of $H=\frac{1}{2}$.

Remark 4 One can envision that the strong Piterbarg property can be applied to functionals $\Phi: C([0, T]) \rightarrow \mathbb{R}$ of $Q_{B_{H}}$ that can be majorized, up to the same magnitude, by the infimum and supremum functionals. A simple example is the integral functional. Theorem 1 yields, for every $H>\frac{1}{2}$,

$$
\mathbb{P}\left(\int_{0}^{T(u)} Q_{B_{H}}(t) \mathrm{d} t>u\right) \sim \mathbb{P}\left(Q_{B_{H}}(0)>\frac{u}{T(u)}\right), \quad \text { as } u \rightarrow \infty
$$

for every function $T(u)>0$ such that $T(u)=o\left(u^{\frac{2 H-1}{3 H-1}}\right)$. The problem of the area under the graph of the storage process fed by the Brownian motion, i.e., the case when $H=\frac{1}{2}$, has been considered in Arendarczyk et al. (2013). 


\section{Proof of Lemma 1}

The general idea behind the proof follows the one in Piterbarg (1996), Lemma D.2. For any $u>0$,

$$
\begin{aligned}
& \mathbb{P}\left(\Phi\left(X_{u}\right)>n(u)\right)=\frac{1}{\sqrt{2 \pi}} \int_{\mathbb{R}} \exp \left(-\frac{v^{2}}{2}\right) \mathbb{P}\left(\Phi\left(X_{u}\right)>n(u) \mid X_{u}(\mathbf{0})=v\right) \mathrm{d} v \\
& \sim \Psi(n(u)) \int_{\mathbb{R}} \exp \left(w-\frac{w^{2}}{2 n^{2}(u)}\right) \mathbb{P}\left(\Phi\left(X_{u}\right)>n(u) \mid X_{u}(\mathbf{0})=n(u)-\frac{w}{n(u)}\right) \mathrm{d} w,
\end{aligned}
$$

where we have used the change of variable $v=n(u)-\frac{w}{n(u)}$. Let $\zeta_{u}=\left\{\zeta_{u}(\mathbf{t}): \mathbf{t} \in T\right\}$ be a Gaussian field defined via $\zeta_{u}(\mathbf{t})=n(u)\left(X_{u}(\mathbf{t})-n(u)\right)+w$. Then, using F2, the last integral can be written as

$\int_{\mathbb{R}} \exp \left(w-\frac{w^{2}}{2 n^{2}(u)}\right) \mathbb{P}\left(\Phi\left(\zeta_{u}\right)>w \mid \zeta_{u}(\mathbf{0})=0\right) \mathrm{d} w=\int_{\mathbb{R}} \exp \left(w-\frac{w^{2}}{2 n^{2}(u)}\right) \mathbb{P}\left(\Phi\left(\chi_{u}\right)>w\right) \mathrm{d} w$,

where $\chi_{u}=\left\{\chi_{u}(\mathbf{t}): \mathbf{t} \in T\right\}$ is a Gaussian field defined as $\chi_{u}(\mathbf{t}) \stackrel{\mathrm{d}}{=} \zeta_{u}(\mathbf{t}) \mid \zeta_{u}(\mathbf{0})=0$. For the family of Gaussian distributions that appear inside the integral, for every $\mathbf{t} \in T$,

$$
\begin{aligned}
\mathbb{E} \chi_{u}(\mathbf{t}) & =n(u) \mathbb{E}\left(X_{u}(\mathbf{t}) \mid X_{u}(\mathbf{0})=n(u)-\frac{w}{n(u)}\right)-n^{2}(u)+w \\
& =-n^{2}(u)\left(1-r_{u}(\mathbf{t} ; \mathbf{0})\right)+w\left(1-r_{u}(\mathbf{t} ; \mathbf{0})\right), \\
\mathbb{E} \chi_{u}(\mathbf{0}) & =\mathbb{E} \chi_{u}^{2}(\mathbf{0})=0 .
\end{aligned}
$$

Furthermore, for any $\mathbf{t}_{1}, \mathbf{t}_{2} \in T$,

$$
\begin{aligned}
\operatorname{Var}\left(\chi_{u}\left(\mathbf{t}_{1}\right)-\chi_{u}\left(\mathbf{t}_{2}\right)\right) & =n^{2}(u)\left(\operatorname{Var}\left(\left(X_{u}\left(\mathbf{t}_{1}\right)-X_{u}\left(\mathbf{t}_{2}\right)\right) \mid X_{u}(\mathbf{0})=u-\frac{w}{u}\right)\right) \\
& =2 n^{2}(u)\left(1-r_{u}\left(\mathbf{t}_{1} ; \mathbf{t}_{2}\right)\right)-n^{2}(u)\left(r_{u}\left(\mathbf{t}_{1} ; \mathbf{0}\right)-r_{u}\left(\mathbf{t}_{2} ; \mathbf{0}\right)\right)^{2} .
\end{aligned}
$$

Hence from Eq. 8 it follows that, as $u \rightarrow \infty$, uniformly on $T$,

$$
\begin{aligned}
\mathbb{E} \chi_{u}(\mathbf{t}) & \rightarrow-\sigma_{\eta}^{2}(\mathbf{t}), \\
\operatorname{Var}\left(\chi_{u}\left(\mathbf{t}_{1}\right)-\chi_{u}\left(\mathbf{t}_{2}\right)\right) & \rightarrow 2 \mathbb{V} \operatorname{ar}\left(\eta\left(\mathbf{t}_{1}\right)-\eta\left(\mathbf{t}_{2}\right)\right) .
\end{aligned}
$$

Thus the finite dimensional distributions of $\chi_{u}$ converge to the finite dimensional distributions of $\tilde{\eta}=\left\{\sqrt{2} \eta(\mathbf{t})-\sigma_{\eta}^{2}(\mathbf{t}): \mathbf{t} \in T\right\}$. Therefore $\chi_{u} \stackrel{d}{\rightarrow} \tilde{\eta}$ in $C(T)$, as $u \rightarrow \infty$, provided that the family $\chi=\left\{\chi_{u}: u>0\right\}$ is tight. For this let $\chi_{u}^{\circ}=$ $\left\{\chi_{u}^{\circ}(\mathbf{t}): \mathbf{t} \in T\right\}$ be a centered Gaussian field defined by $\chi_{u}^{\circ}(\mathbf{t})=\chi_{u}(\mathbf{t})-\mathbb{E} \chi_{u}(\mathbf{t})$. In order to prove tightness of the family $\chi=\left\{\chi_{u}: u>0\right\}$ it suffices to show tightness of the centered family $\chi^{\circ}=\left\{\chi_{u}^{\circ}: u>0\right\}$. Since $\chi_{u}^{\circ}(0)=0$ for all $u>0$, then a straightforward consequence of Straf's criterion for tightness of Gaussian fields, Straf (1972), implies that it suffices to show that for any $\mu, \rho>0$, there exists $\delta \in(0,1)$ and $u_{0}>0$ such that, for each $\mathbf{t}_{1} \in T$ and $u>u_{0}$,

$$
\mathbb{P}\left(\sup _{\left\|\mathbf{t}_{1}-\mathbf{t}_{2}\right\| \leq \delta}\left|\chi_{u}^{\circ}\left(\mathbf{t}_{1}\right)-\chi_{u}^{\circ}\left(\mathbf{t}_{2}\right)\right| \geq \mu\right) \leq \rho \delta^{d},
$$


where $\|\mathbf{t}\|=\max \left\{\left|t_{1}\right|, \ldots,\left|t_{d}\right|\right\}$. Note that, for sufficiently large $u$,

$$
\mathbb{E}\left(\chi_{u}^{\circ}\left(\mathbf{t}_{1}\right)-\chi_{u}^{\circ}\left(\mathbf{t}_{2}\right)\right)^{2} \leq C \operatorname{Var}\left(\eta\left(\mathbf{t}_{1}\right)-\eta\left(\mathbf{t}_{2}\right)\right),
$$

for all $\mathbf{t}_{1}, \mathbf{t}_{2} \in T$ and some constant $C>0$. Thus, the assumption $\mathbf{E} 1$ implies,

$$
\sup _{\left\|\mathbf{t}_{1}-\mathbf{t}_{2}\right\| \leq \delta} \operatorname{Var}\left(\chi_{u}^{\circ}\left(\mathbf{t}_{1}\right)-\chi_{u}^{\circ}\left(\mathbf{t}_{2}\right)\right) \leq C G \delta^{\gamma},
$$

which combined with the application of Borell's inequality gives (13).

Then, the continuous mapping theorem implies

$$
\begin{aligned}
\lim _{u \rightarrow \infty} \int_{\mathbb{R}} \exp \left(w-\frac{w^{2}}{2 n^{2}(u)}\right) \mathbb{P}\left(\Phi\left(\chi_{u}\right)>w\right) \mathrm{d} w & =\int_{\mathbb{R}} \exp (w) \mathbb{P}\left(\Phi\left(\sqrt{2} \eta(\cdot)-\sigma_{\eta}^{2}(\cdot)\right)>w\right) \mathrm{d} w \\
& =\mathbb{E} \exp \left(\Phi\left(\sqrt{2} \eta(\cdot)-\sigma_{\eta}^{2}(\cdot)\right)\right)=\mathcal{H}_{\eta}^{\Phi}(T),
\end{aligned}
$$

provided we can interchange the limit with the integral in Eq. 14. From Eq. 8 it follows that $\left(1-r_{u}(\mathbf{t} ; \mathbf{0})\right) \rightarrow 0$ uniformly in $\mathbf{t} \in T$, therefore Eqs. 10-11 imply that for any $\varepsilon>0$ and sufficiently large $u$,

$$
w_{u}:=\sup _{\mathbf{t} \in T} \mathbb{E} \chi_{u}^{\circ}(\mathbf{t}) \leq \varepsilon|w| .
$$

Using Eq. 12 combined with Sudakov-Fernique's inequality yields, for sufficiently large $u$ and some constant $C>0$,

$$
m_{u}:=\mathbb{E} \sup _{\mathbf{t} \in T} \chi_{u}^{\circ}(\mathbf{t}) \leq C \mathbb{E} \sup _{\mathbf{t} \in T} \eta(\mathbf{t})=: m .
$$

Furthermore, Eq. 12 combined with E1 implies, for sufficiently large $u$,

$$
\sigma_{u}^{2}:=\sup _{\mathbf{t} \in T} \operatorname{Var}\left(\chi_{u}^{\circ}(\mathbf{t})\right) \leq C \sup _{\mathbf{t} \in T} \sigma_{\eta}^{2}(\mathbf{t}) \leq C G(\operatorname{diam}(T))^{\gamma} .
$$

Now, by F1, Borell's inequality yields, for $|w|(1-\varepsilon) \geq m$,

$$
\begin{aligned}
\mathbb{P}\left(\Phi\left(\chi_{u}\right)>w\right) & \leq \mathbb{P}\left(\sup _{\mathbf{t} \in T} \chi_{u}^{\circ}(\mathbf{t})>w-w_{u}\right) \leq \mathbb{P}\left(\sup _{\mathbf{t} \in T} \chi_{u}^{\circ}(\mathbf{t})-m_{u}>w-\varepsilon|w|-m_{u}\right) \\
& \leq 2 \exp \left(-\frac{\left(w-\varepsilon|w|-m_{u}\right)^{2}}{2 \sigma_{u}^{2}}\right) \leq 2 \exp \left(-\frac{(w-\varepsilon|w|-m)^{2}}{2 C G(\operatorname{diam}(T))^{\gamma}}\right) .
\end{aligned}
$$

Hence the interchange of the limit with the integral in Eq. 14 follows by the dominated convergence theorem and the limit is finite, that is $\mathcal{H}_{\eta}^{\Phi}(T)<\infty$.

This completes the proof of Lemma 1.

\section{Proof of Theorem 1}

We divide the proof on a number of steps. Before we proceed, let us make the following observation. The time-reversibility property of $\mathrm{fBm}$ implies that (on the process level)

$$
Q_{B_{H}}(t) \stackrel{\mathrm{d}}{=} \sup _{\sigma \geq t}\left(B_{H}(\sigma)-B_{H}(t)-c(\sigma-t)\right),
$$

which is the form of $Q_{B_{H}}$ that we shall use in this section. The relations of Sections 6.1 and 6.2 were derived in Piterbarg (2001). 


\subsection{Reduction to a Gaussian field}

Using new variables $\tau=(\sigma-t) / u$ and $s=t / u$, for any $T>0$,

$$
\begin{aligned}
& \mathbb{P}\left(\inf _{t \in[0, T]} Q_{B_{H}}(t)>u\right)=\mathbb{P}\left(\inf _{t \in[0, T]} \sup _{\sigma \geq t}\left(B_{H}(\sigma)-B_{H}(t)-c(\sigma-t)\right)>u\right) \\
& =\mathbb{P}\left(\forall s \in\left[0, \frac{T}{u}\right] \exists \tau \geq 0: B_{H}(u(s+\tau))-B_{H}(s u)>u+c u \tau\right) \\
& =\mathbb{P}\left(\inf _{s \in\left[0, T u^{-1}\right]} \sup _{\tau \geq 0} \frac{B_{H}(u(s+\tau))-B_{H}(s u)}{\tau^{H} u^{H} v(\tau)}>u^{1-H}\right) \\
& =\mathbb{P}\left(\inf _{s \in\left[0, T u^{-1}\right]} \sup _{\tau \geq 0} Z_{u}(s, \tau)>u^{1-H}\right),
\end{aligned}
$$

where $v(\tau)=\tau^{-H}+c \tau^{1-H}$ and $Z_{u}=\left\{Z_{u}(s, \tau): s, \tau \geq 0\right\}$ is a Gaussian field given by

$$
Z_{u}(s, \tau)=\frac{B_{H}(u(s+\tau))-B_{H}(s u)}{\tau^{H} u^{H} v(\tau)} .
$$

The distribution of $Z_{u}$ does not depend on $u$, hence we deal with $Z=Z_{1}$. Note that $Z(s, \tau)$ is stationary in $s$, but not in $\tau$.

\subsection{Correlation structure of $Z$}

The variance $\sigma_{Z}^{2}(\tau)$ of $Z(s, \tau)$ equals $v^{-2}(\tau)$ and has a single maximum point at $\tau_{0}=\frac{H}{c(1-H)}$. Taylor expansion shows that, as $\tau \rightarrow \tau_{0}$,

$$
\sigma_{Z}(\tau)=\frac{1}{A}-\frac{B}{2 A^{2}}\left(\tau-\tau_{0}\right)^{2}+O\left(\left(\tau-\tau_{0}\right)^{3}\right),
$$

where

$$
\begin{aligned}
& A=\frac{1}{1-H}\left(\frac{H}{c(1-H)}\right)^{-H}=v\left(\tau_{0}\right), \\
& B=H\left(\frac{H}{c(1-H)}\right)^{-H-2}=v^{\prime \prime}\left(\tau_{0}\right) .
\end{aligned}
$$

Furthermore, denote $a=\frac{1}{2} \tau_{0}^{-2 H}$ and $b=\frac{B}{2 A}$. Note that $\tau_{0}, A, B, a, b$ are the same constants as in Section 4.

The correlation function $r\left(s_{1}, \tau_{1} ; s_{2}, \tau_{2}\right)$ of $Z$ equals

$$
\begin{aligned}
& r\left(s_{1}, \tau_{1} ; s_{2}, \tau_{2}\right)=\mathbb{E} Z\left(s_{1}, \tau_{1}\right) Z\left(s_{2}, \tau_{2}\right) \nu\left(\tau_{1}\right) \nu\left(\tau_{2}\right) \\
& =\frac{\left|s_{1}-s_{2}+\tau_{1}\right|^{2 H}+\left|s_{1}-s_{2}-\tau_{2}\right|^{2 H}-\left|s_{1}-s_{2}+\tau_{1}-\tau_{2}\right|^{2 H}-\left|s_{1}-s_{2}\right|^{2 H}}{2 \tau_{1}^{H} \tau_{2}^{H}} \\
& =1-a(1+o(1))\left(\left|s_{1}-s_{2}+\tau_{1}-\tau_{2}\right|^{2 H}+\left|s_{1}-s_{2}\right|^{2 H}\right)
\end{aligned}
$$

as $s_{1}-s_{2} \rightarrow 0, \tau_{1} \rightarrow \tau_{0}, \tau_{2} \rightarrow \tau_{0}$. 


\subsection{Asymptotic properties of $Z$}

In this step we will be concerned with the asymptotic properties of

$$
\mathbb{P}\left(\inf _{s \in[0, T]} \sup _{\tau \geq 0} A Z(s, \tau)>u\right)
$$

as $u$ grows to infinity. Note that we normalized $Z$ such that now the variance of $A Z(s, \tau)$ equals one at $\tau=\tau_{0}(Z$ is stationary in $s)$. It follows from [Piterbarg 2001, Lemma 1] that there exists a constant $C$ such that, for any $T>0$ and sufficiently large $u$,

$$
\mathbb{P}\left(\inf _{s \in[0, T]} \sup _{\left|\tau-\tau_{0}\right| \geq \log u / u} A Z(s, \tau)>u\right) \leq C T u^{2 / H} \exp \left(-\frac{1}{2} u^{2}-b \log ^{2} u\right) .
$$

If we restrict ourselves to the neighborhood $\left\{\tau:\left|\tau-\tau_{0}\right| \leq \log u / u\right\}$ of $\tau_{0}$, then the following step shows that the probability in Eq. 17, with $Z$ restricted to the neighborhood of $\tau_{0}$, on the logarithmic scale decays as $-\frac{u^{2}}{2}$ when $u$ grows large. Therefore, the neighborhood of $\tau_{0}$ has the largest contribution to the asymptotic behavior of Eq. 17. In the following step we present its asymptotic contribution.

\subsection{The asymptotics of the main contributor}

In this step we show that for any $\lambda>0$, with $\mathcal{H}_{B_{H}}^{\text {sup }}$ defined in Eq. 9,

$$
\liminf _{u \rightarrow \infty} \frac{\mathbb{P}\left(\inf _{s \in\left[0, \lambda u^{-1 / H}\right]} \sup _{\left|\tau-\tau_{0}\right| \leq \log u / u} A Z(s, \tau)>u\right)}{\sqrt{\pi} a^{\frac{1}{2 H}} b^{-\frac{1}{2}} \mathcal{H}_{B_{H}}^{\sup _{H}} \mathcal{H}_{B_{H}}^{\inf }\left(\left[0, \lambda a^{\frac{1}{2 H}}\right]\right) u^{\frac{1}{H}-1} \Psi(u)} \geq 1 .
$$

For the Gaussian field $X(s, \tau)=A Z(s, \tau-s)$, we have

$$
\mathbb{P}\left(\inf _{s \in\left[0, \lambda u^{-\frac{1}{H}}\right]\left|\tau-\tau_{0}\right| \leq \frac{\log u}{u}} A Z(s, \tau)>u\right) \geq \mathbb{P}\left(\inf _{s \in\left[0, \lambda u^{-\frac{1}{H}}\right]} \sup _{\tau \in I} X(s, \tau)>u\right),
$$

for sufficiently large $u$, where $I:=\left[\tau_{0}-\frac{\log u}{2 u}, \tau_{0}+\frac{\log u}{2 u}\right]$ (we use that $I \subset$ $\left[\tau_{0}+s-\frac{\log u}{u}, \tau_{0}+s+\frac{\log u}{u}\right]$ for sufficiently large $\left.u\right)$. From Eq. 16 it follows that the correlation function $r_{X}$ of $X$ is given by

$$
r_{X}\left(s_{1}, \tau_{1} ; s_{2}, \tau_{2}\right)=1-a(1+o(1))\left(\left|\tau_{1}-\tau_{2}\right|^{2 H}+\left|s_{1}-s_{2}\right|^{2 H}\right)
$$

as $s_{1}-s_{2} \rightarrow 0, \tau_{1}-s_{1} \rightarrow \tau_{0}, \tau_{2}-s_{2} \rightarrow \tau_{0}$. Furthermore, Eq. 15 implies that the variance function $\sigma_{X}^{2}$ of $X$ satisfies

$$
\sigma_{X}(s, \tau)=1-b\left(\tau-s-\tau_{0}\right)^{2}+O\left(\left(\tau-s-\tau_{0}\right)^{3}\right),
$$

as $\tau-s \rightarrow \tau_{0}$. 
Let us divide the interval $\left[\tau_{0}-\frac{\log u}{2 u}, \tau_{0}+\frac{\log u}{2 u}\right]$ into intervals of length $\gamma u^{-\frac{1}{H}}$ for some fixed $\gamma>0$,

$$
\begin{array}{cl}
I_{k} & =\left[\tau_{0}+k \gamma u^{-\frac{1}{H}}, \tau_{0}+(k+1) \gamma u^{-\frac{1}{H}}\right], \quad k=0,1,2, \ldots, \\
I_{-k} & =\left[\tau_{0}-(k+1) \gamma u^{-\frac{1}{H}}, \tau_{0}-k \gamma u^{-\frac{1}{H}}\right], \quad k=0,1,2, \ldots,
\end{array}
$$

Notice that,

$$
\begin{aligned}
& \mathbb{P}\left(\inf _{s \in\left[0, \lambda u^{-\frac{1}{H}}\right]} \sup _{\tau \in I} X(s, \tau)>u\right)
\end{aligned}
$$

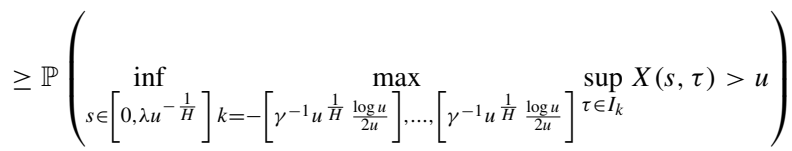

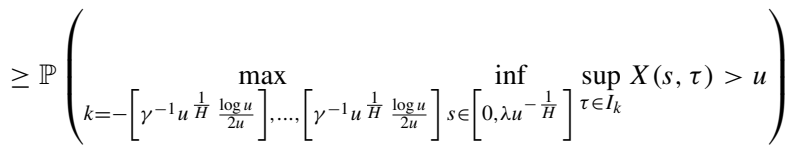

$$
\begin{aligned}
& \geq 2 \sum_{k=0}^{\left[\gamma^{-1} u^{\frac{1}{H}} \frac{\log u}{2 u}\right]} \mathbb{P}\left(\inf _{s \in\left[0, \lambda u^{-\frac{1}{H}}\right]} \sup _{\tau \in I_{k}} X(s, \tau)>u\right) \\
& -2 \sum_{0 \leq l<k \leq\left[\gamma^{-1} u^{\frac{1}{H}} \frac{\log u}{2 u}\right]} \mathbb{P}\left(\inf _{s \in\left[0, \lambda u^{-\frac{1}{H}}\right]} \sup _{\tau \in I_{k}} X(s, \tau)>u, \inf _{s \in\left[0, \lambda u^{-\frac{1}{H}}\right]} \sup _{\tau \in I_{l}} X(s, \tau)>u\right) \\
& -\mathbb{P}\left(\inf _{s \in\left[0, \lambda u^{-\frac{1}{H}}\right]} \sup _{\tau \in I_{-0}} X(s, \tau)>u, \inf _{s \in\left[0, \lambda u^{-\frac{1}{H}}\right]} \sup _{\tau \in I_{0}} X(s, \tau)>u\right) .
\end{aligned}
$$

Now, for any $\varepsilon>0$, any $s \in\left[0, \lambda u^{-\frac{1}{H}}\right]$ and all $\tau \in I_{ \pm k}$, for sufficiently large $u$,

$$
1-(b+\varepsilon)(k+1)^{2} \gamma^{2} u^{-\frac{2}{H}} \leq \sigma_{X}(s, \tau) \leq 1-b(1-\varepsilon) k^{2} \gamma^{2} u^{-\frac{2}{H}} .
$$

Therefore, with $\bar{X}(s, \tau)=X(s, \tau) / \sigma_{X}(s, \tau)$,

$$
\mathbb{P}\left(\inf _{s \in\left[0, \lambda u^{-\frac{1}{H}}\right]} \sup _{\tau \in I_{k}} X(s, \tau)>u\right) \geq \mathbb{P}\left(\inf _{s \in\left[0, \lambda u^{-\frac{1}{H}}\right]} \sup _{\tau \in I_{k}} \bar{X}(s, \tau)>u_{k+}\right),
$$

where

$$
u_{k+}=\frac{u}{1-(b+\varepsilon)(k+1)^{2} \gamma^{2} u^{-\frac{2}{H}}} .
$$


Thus by Example 1, as $u \rightarrow \infty$,

$$
\begin{aligned}
& 2 \sum_{k=0}^{\left[\gamma^{-1} u^{\frac{1}{H}} \frac{\log u}{2 u}\right]} \mathbb{P}\left(\inf _{s \in\left[0, \lambda u^{-\frac{1}{H}}\right]} \sup _{\tau \in I_{k}} X(s, \tau)>u\right) \\
& \geq 2(1+o(1)) \sum_{k=0}^{\left[\gamma^{-1} u^{\frac{1}{H}} \frac{\log u}{u}\right]} \mathcal{H}_{B_{H}}^{\inf }\left(\left[0, \lambda a^{\frac{1}{2 H}}\right]\right) \mathcal{H}_{B_{H}}^{\sup }\left(\left[0, \gamma a^{\frac{1}{2 H}}\right]\right) \Psi\left(u_{k_{+}}\right) .
\end{aligned}
$$

Notice that (cf. Eq. 6), as $u \rightarrow \infty$,

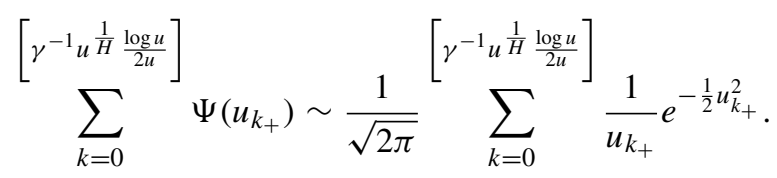

Furthermore, as $u \rightarrow \infty$,

$$
\begin{aligned}
& \frac{1}{\sqrt{2 \pi}} \sum_{k=0}^{\left[\gamma^{-1} u^{\frac{1}{H}} \frac{\log u}{2 u}\right]} \frac{1}{u_{k_{+}}} e^{-\frac{1}{2} u_{k_{+}}^{2}}=\frac{1}{u \sqrt{2 \pi}} \sum_{k=0}^{\left[\gamma^{-1} u^{\frac{1}{H}} \frac{\log u}{2 u}\right]}\left(1-(b+\varepsilon)(k+1)^{2} \gamma^{2} u^{-\frac{2}{H}}\right) \\
& \times \exp \left(\frac{-u^{2}}{2\left(1-(b+\varepsilon)(k+1)^{2} \gamma^{2} u^{-\frac{2}{H}}\right)^{2}}\right) \\
& =\frac{1}{u \sqrt{2 \pi}} \sum_{k=0}^{\left[\gamma^{-1} u^{\frac{1}{H}} \frac{\log u}{2 u}\right]} \exp \left(\frac{-u^{2}}{2\left(1-(b+\varepsilon)(k+1)^{2} \gamma^{2} u^{-\frac{2}{H}}\right)^{2}}\right)(1+o(1))
\end{aligned}
$$

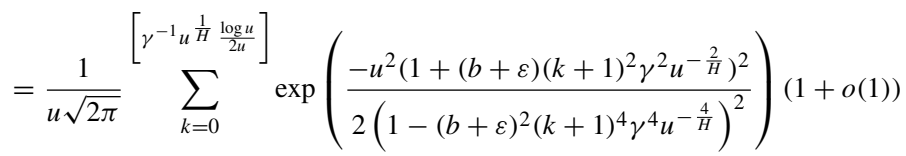

$$
\begin{aligned}
& =\frac{1}{u \sqrt{2 \pi}} \exp \left(-\frac{u^{2}}{2}\right)^{\left[\gamma^{-1} u^{\frac{1}{H} \frac{\log u}{2 u}}\right]} \sum_{k=0} \exp \left(\frac{-u^{2}(b+\varepsilon)(k+1)^{2} \gamma^{2} u^{-\frac{2}{H}}}{\left(1-(b+\varepsilon)^{2}(k+1)^{4} \gamma^{4} u^{-\frac{4}{H}}\right)^{2}}\right)(1+o(1)) \\
& =\Psi(u) \sum_{k=0}^{\left[\gamma^{-1} u^{\frac{1}{H}} \frac{\log u}{2 u}\right]} \exp \left(-(b+\varepsilon)(k+1)^{2} \gamma^{2} u^{2-\frac{2}{H}}\right)(1+o(1)) \\
& =\Psi(u) u^{\frac{1}{H}-1} \sum_{k=0}^{\left[\gamma^{-1} u^{\frac{1}{H} \frac{\log u}{2 u}}\right]} u^{1-\frac{1}{H}} \exp \left(-(b+\varepsilon) \gamma^{2}\left((k+1) u^{1-\frac{1}{H}}\right)^{2}\right)(1+o(1)) \\
& =\Psi(u) u^{\frac{1}{H}-1} \int_{0}^{\infty} \exp \left(-(b+\varepsilon) \gamma^{2} x^{2}\right) \mathrm{d} x(1+o(1))
\end{aligned}
$$

and

$$
\int_{0}^{\infty} \exp \left(-(b+\varepsilon) \gamma^{2} x^{2}\right) \mathrm{d} x=\frac{\sqrt{\pi}}{2 \gamma \sqrt{b+\varepsilon}}
$$


Combining these estimates we obtain

$$
\begin{aligned}
& 2 \sum_{k=0}^{\left[\gamma^{-1} u^{\frac{1}{H}} \frac{\log u}{2 u}\right]} \mathbb{P}\left(\operatorname { i n f } _ { s \in [ 0 , \lambda u ^ { - \frac { 1 } { H } } ] \operatorname { s u p } _ { \tau \in I _ { k } } X ( s , \tau ) > u ) } \left(\mathcal{H}_{B_{H}}^{\inf }\left(\left[0, \lambda a^{\frac{1}{2 H}}\right]\right) \mathcal{H}_{B_{H}}^{\sup }\left(\left[0, \gamma a^{\frac{1}{2 H}}\right]\right) \Psi(u) u^{\frac{1}{H}-1} \frac{\sqrt{\pi}}{2 \gamma \sqrt{b+\varepsilon}}(1+o(1)),\right.\right.
\end{aligned}
$$

which, by the fact that $\varepsilon, \gamma>0$ were arbitrary and $\lim _{S \rightarrow \infty} \frac{1}{S} \mathcal{H}_{B_{H}}^{\text {sup }}([0, S])=\mathcal{H}_{B_{H}}^{\text {sup }}$, yield

$$
2 \sum_{k=0}^{\left[\gamma^{-1} u^{\frac{1}{H}} \frac{\log u}{2 u}\right]} \mathbb{P}\left(\inf _{s \in\left[0, \lambda u^{-\frac{1}{H}}\right]} \sup _{\tau \in I_{k}} X(s, \tau)>u\right) \geq \mathcal{H}_{B_{H}}^{\inf }\left(\left[0, \lambda a^{\frac{1}{2 H}}\right]\right) \mathcal{H}_{B_{H}}^{\text {sup }} a^{\frac{1}{2 H}} \frac{\sqrt{\pi}}{\sqrt{b}} u^{\frac{1}{H}-1} \Psi(u)(1+o(1)) .
$$

Finally, note that

$$
\begin{aligned}
& 2 \sum_{0 \leq l<k \leq\left[\gamma^{-1} u^{\frac{1}{H}} \frac{\log u}{2 u}\right]} \mathbb{P}\left(\inf _{s \in\left[0, \lambda u^{-\frac{1}{H}}\right]} \sup _{\tau \in I_{k}} X(s, \tau)>u, \inf _{s \in\left[0, \lambda u^{-\frac{1}{H}}\right]} \sup _{\tau \in I_{l}} X(s, \tau)>u\right) \\
& +\mathbb{P}\left(\inf _{s \in\left[0, \lambda u^{-\frac{1}{H}}\right]} \sup _{\tau \in I_{-0}} X(s, \tau)>u, \inf _{s \in\left[0, \lambda u^{-\frac{1}{H}}\right]} \sup _{\tau \in I_{0}} X(s, \tau)>u\right) \\
& \leq 2 \sum_{0 \leq l<k \leq\left[\gamma^{-1} u^{\left.\frac{1}{H} \frac{\log u}{2 u}\right]}\right.} \mathbb{P}\left(\sup _{s \in\left[0, \lambda u^{-\frac{1}{H}}\right]^{\tau \in I_{k}}} X(s, \tau)>u, \sup _{s \in\left[0, \lambda u^{-\frac{1}{H}}\right]^{\tau \in I_{l}}} X(s, \tau)>u\right)
\end{aligned}
$$

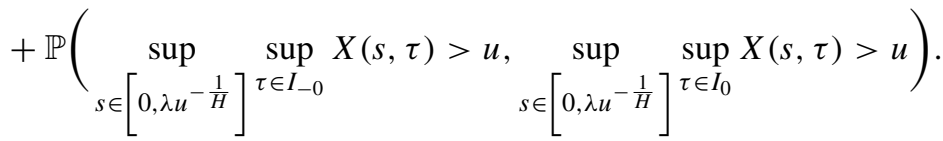

It has been shown in (Piterbarg 2001 end of the proof of Lemma 3), that the last expression is of a smaller order than $u^{\frac{1}{H}-1} \Psi(u)$, which completes the proof of this step.

\subsection{Derivation of the asymptotics}

Recall from Section 6.1 that, for any $T>0$,

$$
P(u):=\mathbb{P}\left(\inf _{t \in[0, T]} Q_{B_{H}}(t)>u\right)=\mathbb{P}\left(\inf _{s \in\left[0, T A^{\frac{1}{1-H}}\left(A u^{1-H}\right)^{-\frac{1}{1-H}}\right]}^{\sup _{\tau \geq 0} A Z(s, \tau)>A u^{1-H}}\right) .
$$


Theorem 1 is a simple reformulation of the observations of the previous steps in terms of the storage process $Q_{B_{H}}$. We have,

$$
\left[0, T A^{\frac{1}{1-H}}\left(A u^{1-H}\right)^{-\frac{1}{1-H}}\right]=\left[0, \lambda(u)\left(A u^{1-H}\right)^{-\frac{1}{H}}\right],
$$

where $\lambda(u)=T A^{\frac{1}{H}} u^{\frac{1-2 H}{H}}$. Let $T=T(u)$ be such that $T(u)=o\left(u^{\frac{2 H-1}{H}}\right)$ as $u \rightarrow$ $\infty$. Then, for any $\varepsilon>0$ and all $u$ such that $\lambda(u) \leq \varepsilon$,

$$
\left[0, T A^{\frac{1}{1-H}}\left(A u^{1-H}\right)^{-\frac{1}{1-H}}\right] \subset\left[0, \varepsilon\left(A u^{1-H}\right)^{-\frac{1}{H}}\right] .
$$

Hence,

$$
P(u) \geq \mathbb{P}\left(\inf _{s \in\left[0, \varepsilon\left(A u^{1-H}\right)^{-\frac{1}{H}}\right]\left|\tau-\tau_{0}\right| \leq \log \left(A u^{1-H}\right) /\left(A u^{1-H}\right)} A Z(s, \tau)>A u^{1-H}\right)
$$

and by Eq. 18 the last expression is asymptotically bounded below by

$$
\sqrt{\pi} a^{\frac{1}{2 H}} b^{-\frac{1}{2}} \mathcal{H}_{B_{H}}^{\sup _{H}} \mathcal{H}_{B_{H}}^{\inf }\left(\left[0, \varepsilon a^{\frac{1}{2 H}}\right]\right)\left(A u^{1-H}\right)^{\frac{1}{H}-1} \Psi\left(A u^{1-H}\right) .
$$

Observe that by Fatou's lemma lim $\sup _{\varepsilon} \downarrow 0 \mathcal{H}_{B_{H}}^{\text {inf }}\left(\left[0, \varepsilon a^{\frac{1}{2 H}}\right]\right)=1$, which implies the appropriate lower bound for $P(u)$. Finally, recall from Eq. 1, that

$$
\mathbb{P}\left(Q_{B_{H}}(0)>u\right) \sim \sqrt{\pi} a^{\frac{1}{2 H}} b^{-\frac{1}{2}} \mathcal{H}_{B_{H}}^{\text {sup }} \cdot\left(A u^{1-H}\right)^{\frac{1-H}{H}} \Psi\left(A u^{1-H}\right) \text {, as } u \rightarrow \infty,
$$

which is the upper bound for $P(u)$. This completes the proof of Theorem 1 .

Acknowledgments K. Dȩbicki was partially supported by NCN Grant No 2013/09/B/ST1/01778 (20142016) and by the project RARE -318984, a Marie Curie FP7 IRSES Fellowship.

Open Access This article is distributed under the terms of the Creative Commons Attribution License which permits any use, distribution, and reproduction in any medium, provided the original author(s) and the source are credited.

\section{References}

Adler, R.J.: An Introduction to Continuity, Extrema, and Related Topics for General Gaussian Processes, volume 12 of Lecture Notes-Monograph Series. IMS (1990)

Albin, J., Samorodnitsky, G.: On overload in a storage model, with a self-similar and infinitely divisible input. Ann. Appl. Probab. 14, 820-844 (2004)

Arendarczyk, M., DȨbicki, K., Mandjes, M.: On the Tail Asymptotics of the Area Swept under the Brownian Storage Graph. Bernoulli (2013)

Asmussen, S.: Applied Probability and Queues, 2nd edn. Springer (2003)

Asmussen, S., Albrecher, H.: Ruin Probabilities, 2nd edn. World Scientific Publishing Co. Inc. (2010)

Dȩbicki, K.: Ruin probability for Gaussian integrated processes. Stoch. Process Appl. 98, 151-174 (2002)

Dębicki, K., Kisowski, P.: Asymptotics of supremum distribution of $(a(t), a(t))$-locally stationary Gaussian processes. Stoch. Process Appl. 118, 2022-2037 (2008) 
Dębicki, K., Tabiś, K.: Extremes of time-average stationary Gaussian processes. Stoch. Process Appl. 121, 2049-2063 (2011)

Dębicki, K., Kosiński, K.M., Mandjes, M.: On the infimum attained by a reflected Lévy process. Queueing Syst. 70, 23-35 (2012)

Hashorva, E., Ji, L., Piterbarg, V.I.: On the supremum of $\gamma$-reflected processes with fractional Brownian motion as input. Stochastic Process. Appl. (2013). in press

Hüsler, J., Piterbarg, V.I.: Extremes of a certain class of Gaussian processes. Stoch. Process Appl. 83, 257271 (1999)

Hüsler, J., Piterbarg, V.I.: On the ruin probability for physical fractional brownian motion. Stoch. Process Appl. 113, 315-332 (2004)

Mikosch, T., Resnick, S., Rootzén, H., Stegeman, A.: Is network traffic approximated by stable Lévy motion or fractional Brownian motion. Ann. Appl. Probab. 12, 23-68 (2002)

Norros, I.: A storage model with selfsimilar input. Queueing Syst. 16, 387-396 (2004)

Pickands, J. III.: Asymptotic properties of the maximum in a stationary Gaussian process. Trans. Amer. Math. Soc. 145, 75-86 (1969a)

Pickands, J. III.: Upcrossing probabilities for stationary Gaussian processes. Trans. Amer. Math. Soc. 145, 51-73 (1969b)

Piterbarg, V.I.: Asymptotics Methods in the Theory of Gaussian Processes and Fields, volume 148 of Translation of Mathematical Monographs. AMS (1996)

Piterbarg, V.I.: Large deviations of a storage process with fractional Brownian motion as input. Extremes 4, 147-164 (2001)

Piterbarg, V.I., Prisyazhnyuk, V.: Asymptotic behavior of the probability of a large excursion for a nonstationary gaussian processes. Theory Probab. Math. Stat., 121-133 (1978)

Reich, E.: On the integrodifferential equation of Takács I. Ann. Math. Stat. 29, 563-570 (1958)

Straf, M.L.: Weak convergence of stochastic processes with several parameters. In: Proceedings of the Sixthe Berkeley Symposium in Mathematical Statistics and Probability, vol. 2, pp. 187-221 (1972)

Taqqu, M.S., Willinger, W., Sherman, R.: Proof of a fundamental result in self-similar traffic modeling. Comput. Comm. Rev. 27, 5-23 (1997) 